\title{
Yersinia enterocolitica palearctica serobiotype 0:3/4 - a successful group of emerging zoonotic pathogens
}

\author{
Julia Batzilla', Uladzimir Antonenka', Dirk Höper², Jürgen Heesemann and Alexander Rakin ${ }^{1 *}$
}

\begin{abstract}
Background: High-pathogenic Y. enterocolitica ssp. enterocolitica caused several human outbreaks in Northern America. In contrast, low pathogenic $Y$. enterocolitica ssp. palearctica serobiotype 0:3/4 is responsible for sporadic cases worldwide with asymptomatic pigs being the main source of infection. Genomes of three $Y$. enterocolitica ssp. palearctica serobiotype 0:3/4 human isolates (including the completely sequenced Y11 German DSMZ type strain) were compared to the high-pathogenic $Y$. enterocolitica ssp. enterocolitica 8081 0:8/1B to address the peculiarities of the 0:3/4 group.

Results: Most high-pathogenicity-associated determinants of $Y$. enterocolitica ssp. enterocolitica (like the HighPathogenicity Island, yts 1 type 2 and ysa type 3 secretion systems) are absent in Y. enterocolitica ssp. palearctica serobiotype 0:3/4 genomes. On the other hand they possess alternative putative virulence and fitness factors, such as a different ysp type 3 secretion system, an RtxA-like and insecticidal toxins, and a N-acetyl-galactosamine (GalNAc) PTS system (aga-operon). Horizontal acquisition of two prophages and a tRNA-Asn-associated GIYep-01 genomic island might also influence the $Y$. enterocolitica ssp. palearctica serobiotype 0:3/4 pathoadaptation. We demonstrated recombination activity of the PhiYep-3 prophage and the GIYep-01 island and the ability of the agaoperon to support the growth of the Y. enterocolitica ssp. enterocolitica O:8/1B on GalNAc.
\end{abstract}

Conclusions: $Y$. enterocolitica ssp. palearctica serobiotype 0:3/4 experienced a shift to an alternative patchwork of virulence and fitness determinants that might play a significant role in its host pathoadaptation and successful worldwide dissemination.

\section{Background}

The gram-negative bacterium Yersinia enterocolitica is a widely disseminated gastrointestinal pathogen that belongs to the genus Yersinia together with enteropathogenic $Y$. pseudotuberculosis and the plague agent, $Y$. pestis. It has been proposed that $Y$. enterocolitica and $Y$. pseudotuberculosis have diverged within the last 200 million years while $Y$. pestis is a more recent descendant of $Y$. pseudotuberculosis $[1,2]$. All these species have evolved with diverse clinical symptoms. $Y$. pseudotuberculosis can cause tuberculosis-like symptoms in animals. In humans, the clinical manifestations are similar, but often they are more severe compared to those with $Y$. enterocolitica. $Y$. pseudotuberculosis infections can

\footnotetext{
* Correspondence: rakin@mvp.uni-muenchen.de

1 Max von Pettenkofer-Institute, LMU, Munich, Germany

Full list of author information is available at the end of the article
}

mimic appendicitis, mainly in children, and have similar extra intestinal sequelae compared to $Y$. enterocolitica. $Y$. pestis is the agent of the plague, transmitted by the bite of an infected flea and it is primarily a rodent pathogen.

Yersinia enterocolitica can be differentiated by bioand serotyping [3,4]. Biotype (BT) 1A strains are considered as non-pathogenic, whereas high-pathogenic BT1B (predominant in the U.S.A.) and low to moderate-pathogenic BT2-5 (predominant in Europe, Asia and Australia) are enteropathogenic for humans and animals. $Y$. enterocolitica serogroup O:3 biotype 4 (in the following designated as serobiotype O:3/4 or O:3/ 4) comprises about $80-90 \%$ of human isolates in Germany and Europe, with rising global relevance [5-7]. It is responsible for gastroenteritis, lymphadenitis and various extra intestinal sequelae as erythema nodosum
C Biomed Central

() 2011 Batzilla et al; licensee BioMed Central Ltd. This is an Open Access article distributed under the terms of the Creative Commons Attribution License (http://creativecommons.org/licenses/by/2.0), which permits unrestricted use, distribution, and reproduction in any medium, provided the original work is properly cited. 
and reactive arthritis [8]. Asymptomatic and ill pigs are the main animal reservoir of this serobiotype $[9,10]$, leading to a high submission of $\mathrm{O}: 3 / 4$ contaminations in butcher shops in Germany and countries in northeastern Europe [11-14]. In contrast, $Y$. enterocolitica biotype 1B strains (also called New World strains) were documented predominantly in the U.S.A. as human outbreak and environmental isolates. Pathogenicity analysis, however, has been mainly focused on $Y$. enterocolitica serobiotype $\mathrm{O}: 8 / 1 \mathrm{~B}$, of which a complete genome sequence is available (strain 8081, [15], accession no.

\section{NC_008800.1 and}

NC_008791 (plasmid)). The differentiation between biotype $1 \mathrm{~B}$ and the Old World strains has been legitimated in the assembly of two subspecies, $Y$. enterocolitica ssp. enterocolitica for biotype $1 \mathrm{~B}$ and $Y$. enterocolitica ssp. palearctica for the Old World strains [16].

To compare these two groups of geographically and phylogenetically distinct yersiniae, we determined the complete genome sequence of the European serobiotype O:3/4 DSMZ reference strain Y11 isolated from a patient stool (EMBL accession numbers: FR729477 and FR745874 (plasmid) as announced recently [17]) and compared it with the available $Y$. enterocolitica ssp. enterocolitica 8081 O:8/1B genome. Draft sequences of two other $Y$. enterocolitica ssp. palearctica O:3/4 strains of human origin, named Y8265 and Y5307 (derived from a human patient isolate from France and an arthritis positive human patient isolate, respectively) and a closely related $Y$. enterocolitica ssp. palearctica strain (named Y5,27P) of serobiotype O:5,27/3 were used when appropriate to gain a better insight into peculiarities of the $Y$. enterocolitica ssp. palearctica. Comparison of these closely related pathogens enables us to uncover potential pathogenicity and fitness determinants involved in pathoadaptation and worldwide dissemination of $Y$. enterocolitica ssp. palearctica $\mathrm{O}: 3 / 4$.

\section{Results}

\section{General characteristics}

The genome of $Y$. enterocolitica ssp. palearctica Y11 (serobiotype O:3/4) consists of a circular chromosome spanning $4,553,420 \mathrm{bp}$ with a GC content of $47.01 \%$ and a virulence associated pYVO3 plasmid of 72,460 bp with an overall GC content of $43.99 \%$ (see Table 1 and additional file 1). The tabular SEED comparison between serobiotype O:3/4 strains Y11, Y8265, Y5307, serobiotype $0: 5,27 / 3$ and serobiotype $O: 8 / 1 \mathrm{~B}$ strain 8081 is added as additional file 2 .

2. $Y$. enterocolitica ssp. enterocolitica genes absent from $Y$. enterocolitica ssp. palearctica

Certain virulence determinants associated with the high-pathogenicity phenotype of $Y$. enterocolitica ssp. enterocolitica are missing in serobiotype O:3/4. The most prominent are the High-Pathogenicity Island (HPI), involved in the yersiniabactin-mediated iron uptake [18-20], and two chromosomally encoded secretion systems, ysa T3SS and yts1 T2SS, located within the Plasticity Zone (PZ) [21]. Besides HPI and PZ several genomic islands (YGI-2 and YGI-3) and prophages (e.g. ФYE250, ФYE185 (encoding the Vaps virulence-associated proteins)) described for serobiotype O:8/1B strain 8081 are absent in $\mathrm{O}: 3 / 4$. In addition, the pYV plasmids of $\mathrm{O}: 3 / 4$ and $\mathrm{O}: 8 / 1 \mathrm{~B}$ are more divergent than the corresponding genome sequences. In contrast, the pYVe227 plasmid of serobiotype O:9/2 (accession no. AF102990) is closely related to pYVO3 plasmid (additional file 3 ). Also the clusters for the LPS biosynthesis differ. In O:3/4, two separate clusters are present as reported previously (for the outer core, Y11_19901-20011 and O-antigen, Y11_16711-16781) [22,23].

Most of the genome differences between $\mathrm{O}: 8 / 1 \mathrm{~B}$ and other serobiotypes have been described previously [15].

Table 1 General features of the genome and virulence plasmid of serobiotypes 0:3/4 strain Y11 and 0:8/1B strain 8081.

\begin{tabular}{|c|c|c|c|c|}
\hline Feature & Genome Y11 & Genome 8081 & Plasmid pYVO3 Y11 & Plasmid pYVO8 8081 \\
\hline Genome size & 4553420 & 4615899 & 72460 & 67721 \\
\hline GC content in $\%$ & 47.01 & 47.27 & 43.99 & 43 \\
\hline Number of coding sequences (CDS) & 4355 & 3978 & $112^{*}$ & 72 \\
\hline Average size of CDS & 884 & 968 & 506 & 672 \\
\hline Coding density in \% & 85 & 83 & 78 & 71 \\
\hline rRNA clusters & 7 & 7 & none & none \\
\hline tRNAs & 70 & 81 & none & none \\
\hline
\end{tabular}

*) The higher number of CDS for the O:3/4 pYV plasmid compared to O:8/1B is evoked by more transposase-like CDS (including also remnant or fragmented transposases) and more than 20 hypothetical CDS with no strong similarity on protein level to serobiotype O:8/1B. In addition, we found an ars $A B R H$ gene cluster present in the pYV plasmid of serobiotypes $0: 3 / 4$ and $0: 9 / 2$, but not in $0: 8 / 1 \mathrm{~B}$ 
Nevertheless there are some additional potential virulence-associated genes that have been uncovered by our genome comparison, including putative haemolysins (YE2407-2408, YE2966), putative adhesins (YE1873) and a putative ospG-like gene (YE3860).

\section{Y. enterocolitica ssp. palearctica 0:3/4 specific genes absent from $Y$. enterocolitica ssp. enterocolitica 0:8/1B Putative toxins}

In serobiotype $\mathrm{O}: 3 / 4$ we found a gene cluster encoding an RtxA-like protein and its adjacent genes, $r t x C$ and $r t x H$, (Y11_18761, Y11_18771 and Y11_18781) (see Figure 1). The large RtxA protein (Y11_18761, about $350 \mathrm{kDa}$ ) could play a role in the pathogenesis of $\mathrm{O}: 3 / 4$, since members of the wide family of Rtx proteins have been shown to be major contributors to pathogenicity [24-28]. The effector domains in the middle of the protein show no homology to already known family members. Therefore, the potential mode of action of the RtxA-like protein is unclear in this strain. In addition, the genes encoding the RtxA secretion system (Y11_10141-10171) are interrupted by a frame shift in $\mathrm{O}: 3 / 4$, raising the question of functional RtxA export.

Bacteriocins constitute a large group of bacterial toxins used to inhibit growth of closely related bacteria. The bacteriocin Y11_33511 in O:3/4 shows sequence homology to pyocin-like proteins and DNAses, therefore being a potential endonuclease enzyme. It is followed by two putative immunity proteins (Y11_33521-33531). The duplication of immunity protein like genes could hint at a particular toxin with extreme toxicity for its host.

A member of cell wall-associated hydrolases (Y11_03361, a putative invasion-associated protein) of about 422 amino acids is encoded in a $2 \mathrm{kbp}$ large genomic region absent from $\mathrm{O}: 8 / 1 \mathrm{~B}$ strain 8081 . The hydrolase encoding CDS is located between yeiH and yeiE, encoding a potential membrane protein and a

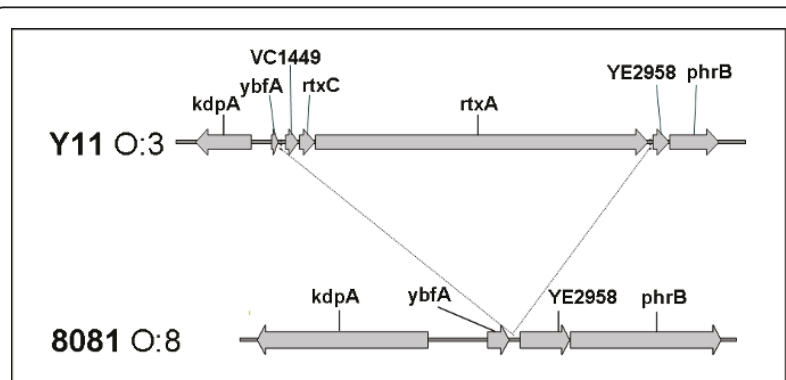

Figure 1 RtxA toxin cluster in $Y$. enterocolitica ssp. palearctica strain Y11 (0:3/4). This gene cluster is inserted into the backbone of $Y$. enterocolitica ssp. enterocolitica strain 8081 (O:8). The cluster comprises $10.6 \mathrm{kbp}$ encoding three genes, $r t \times A, r t \times C$ and a hypothetical VC1449 gene (according to a protein homolog of Vibrio cholerae). transcriptional regulator with yet unknown functions. A 3,075 bp putative invasion precursor gene (Y11_38661) and a $h l y D$ like gene (Y11_09551) have been found in $Y$. enterocolitica $\mathrm{O}: 3 / 4$. The putative invasin revealed homology to Ig-like domains and has a similar domain structure typical of invasion proteins. Genes of the ABC transporter family $([29,30])$ lie adjacent to $h l y D$ in $\mathrm{O}: 3 / 4$ that support its possible export.

We also found several toxin-antitoxin systems in serobiotype $0: 3 / 4$ that are absent or different from those found in serobiotype $\mathrm{O}: 8 / 1 \mathrm{~B}$. One toxin-antitoxin system (TA) was annotated as YgiT-(antitoxin, Y11_40161) and YgiU-(toxin, Y11_40151) like proteins in O:3/4. Kasari et al. reported that the protein YgiU inhibits growth and induces rapid shutdown of protein synthesis in vivo. The cluster is transcriptionally repressed by YgiT and activated by HipA [31]. Another TA cluster found in $\mathrm{O}: 3 / 4$ is annotated as YfjZ (antitoxin, Y11_30951) and YpjF (toxin, Y11_30941), reported to be a putative part of a defective prophage with unknown function [32].

\section{Insecticidal toxin cluster}

An insecticidal toxin cluster (Tc, Y11_26921Y11_27061), initially described in serobiotype O:9/2 [33], is found in serobiotype O:3/4 downstream of tldD, Y11_27071. The toxin cluster comprises four proteins, Tca, Tcb, Tcc and Tcd and is represented in serobiotype $\mathrm{O}: 9 / 2$ by TcaA, TcaB, TcaC and TccC. It was shown that these toxins are active at temperatures below $30^{\circ} \mathrm{C}$ and the lysates could kill $M$. sexta within 5 days [34]. Interestingly, TcaB is split into two proteins and $t c a C$ is interrupted by a stop codon. Still the system was proven to be functional in $0: 9 / 2$. In serobiotype $0: 3 / 4$ one of the two putative tcaA regulatory genes is split into two smaller ones and TcaA (Y11_26951) is a truncated version of the serobiotype O:9/2 protein (see Figure 2). TcaB (Y11_26971) is a single protein in contrast to O:9/ 2 , and the $t c a C^{\prime}(1)$ gene (Y11_26981) undergoes a frame shift at position 3498 (not at position 314 as in $0: 9 / 2$ ). Since this gene cluster differed surprisingly between strains of the same subspecies, we analysed the Tc cluster of the related serobiotype O:5,27/3. This strain also harbours an insecticidal toxin complex closely related to that of serobiotypes $\mathrm{O}: 3 / 4$ and $\mathrm{O}: 9 / 2$. The cluster of $\mathrm{O}: 5,27 / 3$ is the only one with a single $t c a C$ gene (Figure 2 ). In addition, the $t c a R 2$ is, as well as $t c a B$, not fragmented. Another putative insecticidal toxin encoding CDS (Y11_05031), which is not clustered with the Tc cluster described above, is present in O:3/4. The protein is homologous to the insecticidal TccC2 and TccC3 proteins of $Y$. enterocolitica $\mathrm{O}: 9 / 2$.

Flagellar genes, beta-fimbrial genes and other fimbriae A large $21 \mathrm{kbp}$ flagellar cluster (Y11_24071-24361) present in $0: 3 / 4$ genomes is similar to the flag-2 gene 


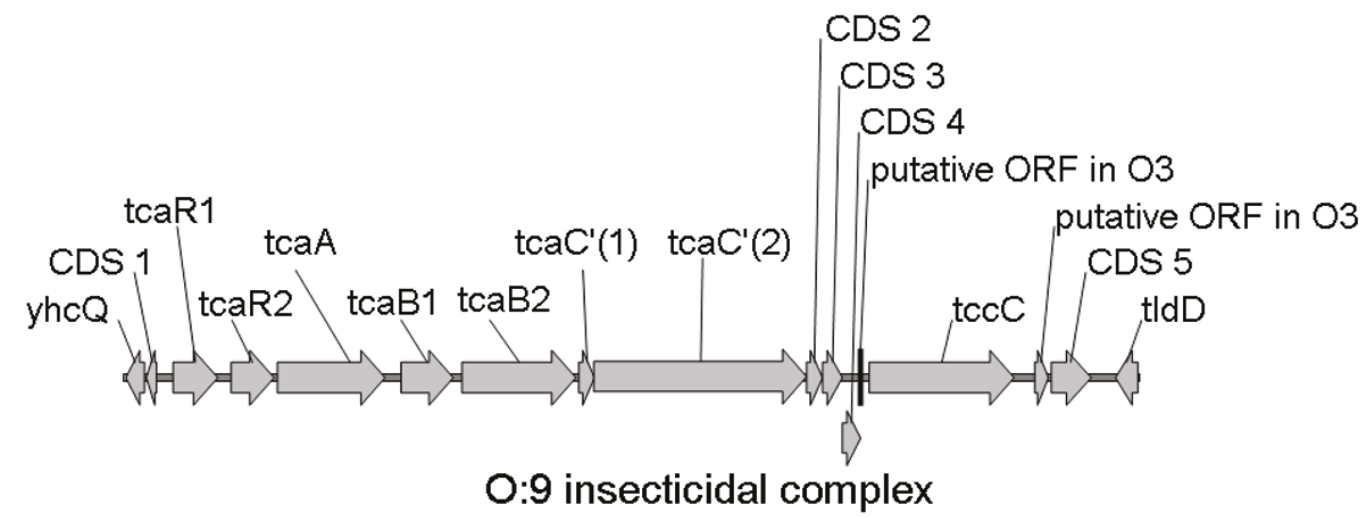

$20,403 \mathrm{bp}$
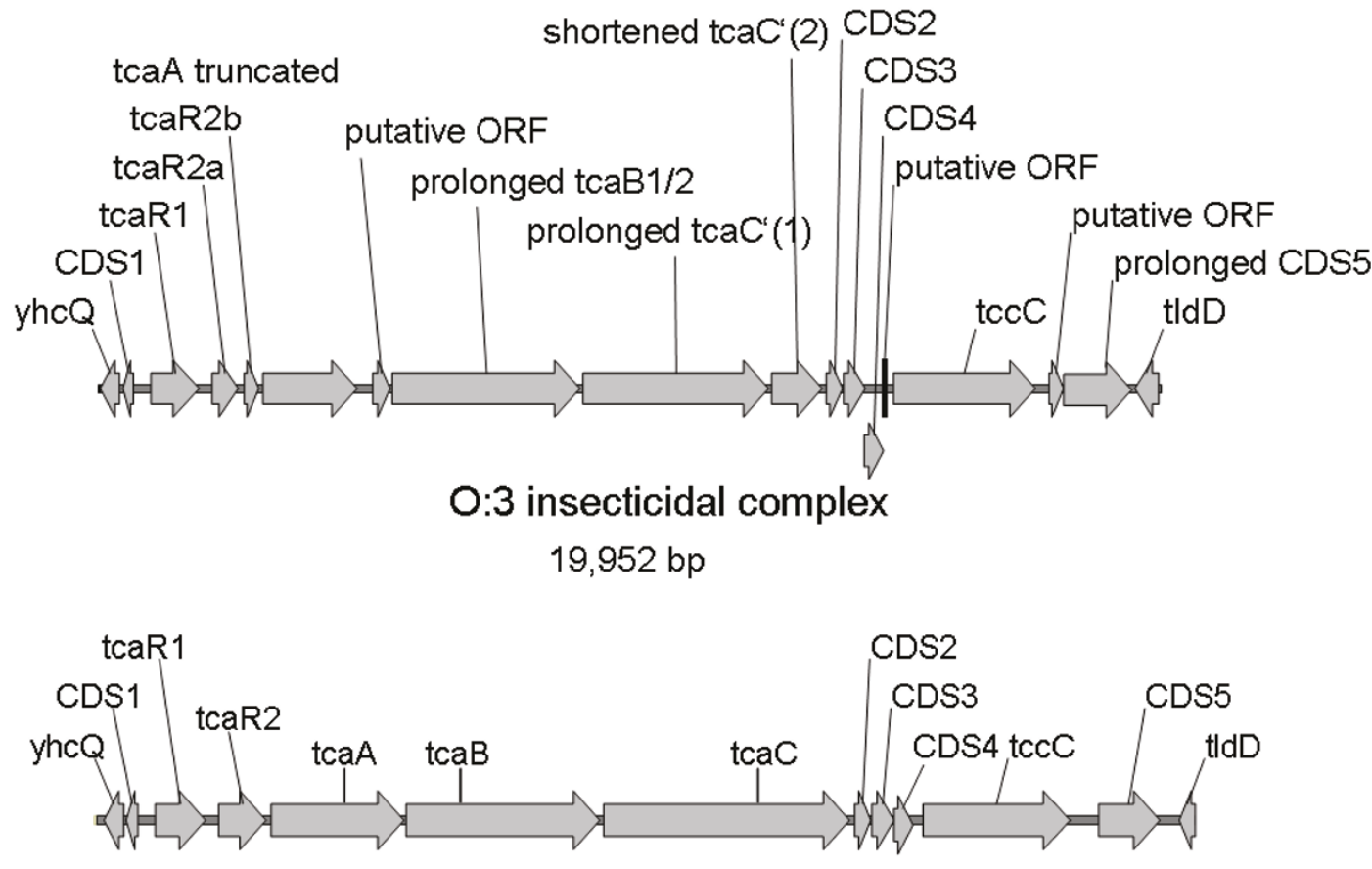

$0: 5 ; 27$ insecticidal complex

$20,049 \mathrm{bp}$

Figure 2 Insecticidal toxin gene complex in $Y$. enterocolitica 0:9/2, 0:3/4 and 0:5,27/3. The structural organisation of the cluster is the same in all serobiotypes, but the encoded proteins differ in length and are to some extent fragmented. For 0:5,27/3, the cluster is the only one with only a single $t c a C$ gene. In addition, the tcaR2 is, as well as $t c a B$, complete. 
cluster of O:9/2 [35]. Only parts of this cluster demonstrated low similarity to O:8/1B genes. The functionality and role of this cluster in pathogenesis are questionable, since experimental observation indicates a weak motility for $\mathrm{O}: 3 / 4$ strains in vitro.

Operons of the $\beta$-fimbriae usually do not resemble typical tip adhesins [36]. They may encode thin fibrillae or nonfimbrial surface structures. We found two clusters of putative beta-fimbriae in O:3/4 (Y11_14931-14971 and Y11_26051-26081), both absent from O:8/1B.

\section{Chromosomally encoded type three secretion system (T3SS) and Aat-secretion}

The virulence-associated chromosomal ysa T3SS is absent in O:3/4. Even so, O:3/4 harbours an alternative 24 kbp T3SS (designated ysp T3SS, Y11_35171-35491, see Figure 3) in the same genome background. The $y s p$ T3SS system is homologous to the Salmonella SPI-2 T3SS [37], but lacks some functional parts. The translocon is present, but the SsaGH proteins of the needle are missing, as well as SsaR from the inner membrane ring. In addition, there are no effector proteins linked directly to the secretion system gene cluster. Thus, the $y s p$ T3SS awaits the identification of its putative effectors and possible involvement in pathogenicity or cell-cell interactions.

The aatPABCD cluster in enteroaggregative $E$. coli (EAEC) encodes a specialized $A B C$ transporter, which plays a role in virulence by transporting dispersin out of bacterial cell [38]. In serobiotype O:3/4, we found a four-gene aat cluster with one gene homologous both to aatB and aatA. The cluster (Y11_24511 -Y11_24421) is interspaced and flanked by small hypothetical genes and transposases. The functionality in $\mathrm{O}: 3 / 4$ is yet

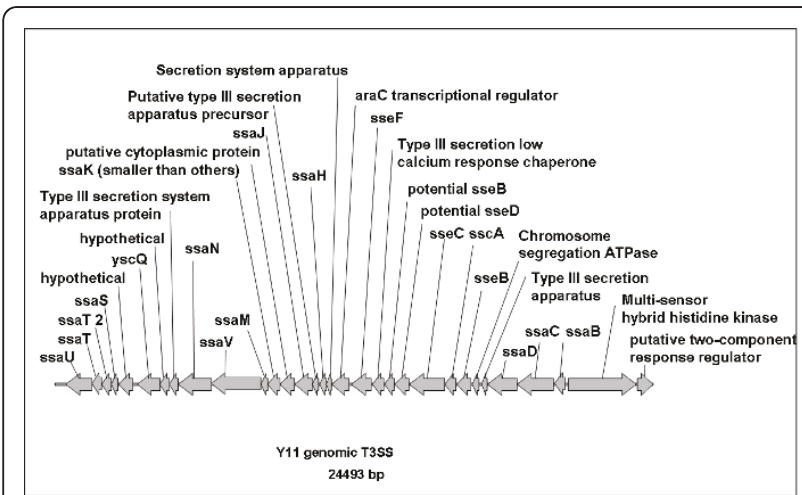

Figure $3 Y$. enterocolitica palearctica 0:3/4-specific chromosomally encoded type three secretion system. This system is similar to the SPI-2 of Salmonella. The translocon of the apparatus is present, but $\mathrm{SsaGH}$ proteins of the needle are missing; also SsaR from the inner membrane ring. In addition, no effector proteins are linked directly to the secretion system gene cluster in the genome strain $\mathrm{Y} 11$. uncharacterised, but a potential role in pathogenesis as for the EAEC, cannot be excluded.

\section{Carbon source uptake and other metabolic differences}

$\mathrm{N}$-acetyl-D-galactosamine and $\mathrm{N}$-acetyl-D-glucosamine are components of the intestinal mucin in pigs and humans. The amount of N-acetyl-D-galactosamine is nearly twice that of any of the other sugars present in the pig's small intestinal mucin [39]. In contrast, Nacetyl-D-glucosamine is the major amino sugar in human mucin [40]. The composition and modification of mucin is a critical defence mechanism in the prevention against pathogenic bacteria in the intestine. Enteric bacteria differ in their ability to grow on N-acetyl-galactosamine (GalNAc or Aga) and on D-galactosamine (GalN or Gam). N-acetyl-galactosamine utilized in Yersinia can be taken up as a carbon source by a specific phosphotransferase system (PTS). The enzymes that build the transport complex are AgaVWEF, and the genes are clustered in an operon in $Y$. enterocolitica $\mathrm{O}: 3 / 4$ (see Figure 4) that is absent in O:8/1B.

The utilisation of different amino sugars (GalNAc, Gam, GlcNAc (N-acetyl-glucosamine) and Nag (D-glucosamine)) was analysed in strains of two serobiotypes, $\mathrm{O}: 3 / 4$ and $\mathrm{O}: 8 / 1 \mathrm{~B}$, in minimal medium with $0.2 \%$ amino sugars. As expected, both serobiotypes were able to grow in $0.2 \% \mathrm{~N}$-acetyl-glucosamine and glucosamine. Since the Gam PTS encoding genes (agaBCD) are absent from serobiotypes $\mathrm{O}: 3 / 4$ and $\mathrm{O}: 8 / 1 \mathrm{~B}$, we found all strains unable to grow in galactosamine as the only source for carbon. However, O:3/4 strain Y11 was able to use GalNAc as the only carbon source, even though we did not find a homolog for the downstream processing aga $A$ gene in O:3/4 (Figure 5). To prove the sufficiency of agaVWEF to support growth on GalNAc, we subcloned the operon (Y11_11961-Y11_12031) into pGEM-T Easy (Promega). Strain WA-314, O:8/1B, transformed with agaVWEF, acquired the ability to grow in the presence of GalNAc. Thus, the aga-operon

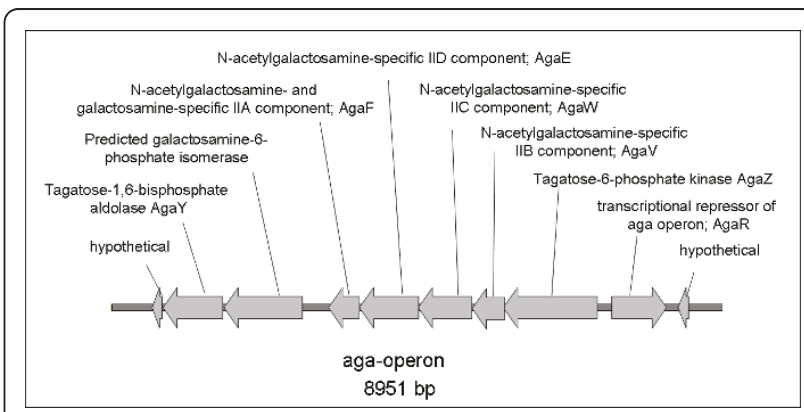

Figure $4 \mathrm{~N}$-acetyl-galactosamine PTS aga-operon (comprising agaVWEF) in $Y$. enterocolitica 0:3/4. Displayed genes have been subcloned into PGEM-T Easy (Promega) to supplement serobiotype $\mathrm{O}: 8 / 1 \mathrm{~B}$ with the additional PTS 


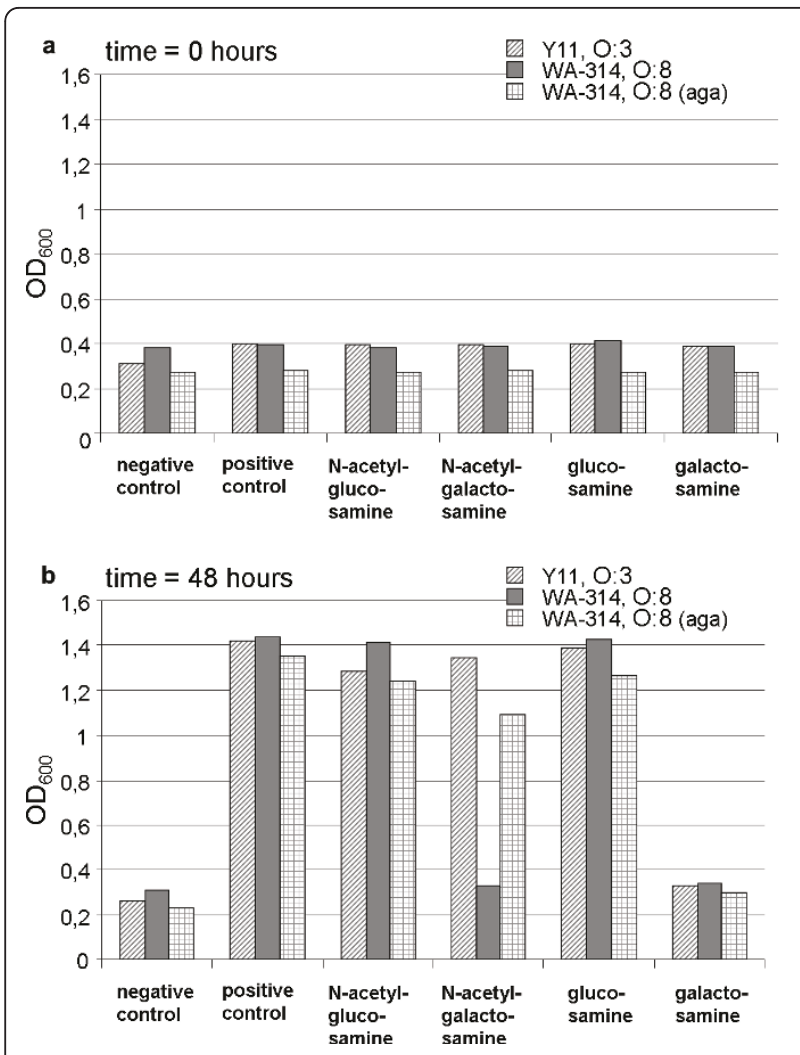

Figure 5 Amino sugar growth experiment. Strains used for the experiments are $\mathrm{Y} 11$ (O:3/4) and WA-314 (O:8/1B) as well as a derivative of WA-314, supplemented with the complete aga-operon (see text). Strains were grown overnight in M9 minimal medium with glucose, washed and inoculated in M9 containing different amino sugars and sugars as the only carbon source. Negative control was M9 without any carbon source, positive control - 0.2\% glucose. a) Measurement of the optical density at $600 \mathrm{~nm}\left(\mathrm{OD}_{600}\right)$ directly after inoculation. b) Measurement of the optical density at $600 \mathrm{~nm}$ after 48 hours of growth at $27^{\circ} \mathrm{C}$.

alone is able to support the utilisation of N-acetyl-galactosamine in vitro and supplies $Y$. enterocolitica with the ability to efficiently utilize this important amino sugar of the intestinal mucin.

We found a second urea transporter system in $\mathrm{O}: 3 / 4$ (Y11_22281-22341), which is independent and different from the first one shared with $\mathrm{O}: 8 / 1 \mathrm{~B}$. There is no obvious explanation for the presence of two different urea clusters in O:3/4. Since the urea systems are unrelated in protein composition, they must have been acquired independently.

Many bacteria isolated from the human gastrointestinal tract show bile salt hydrolase (BSH) activity mediated by the choloylglycine hydrolase (CGH). How this enzyme contributes to the functions of bacteria in the gastrointestinal tract is not known. Studies have shown that choloylglycine hydrolase (CGH) confers the ability to resist the antimicrobial action of bile salts [41].
Therefore, the CGH may contribute to the ability of bacteria to infect the host through the oral route. We found one CGH in O:3/4 (Y11_23571). The gene for $\mathrm{CGH}$ is absent from $\mathrm{O}: 8 / 1 \mathrm{~B}$, reflecting different niches and host infection routes.

\section{Mobile elements shaping $Y$. enterocolitica ssp. palearctica genome Genomic Islands}

Mobile genetic elements are known to be involved in horizontal gene transfer, HGT. They utilize site-specific integrases for recombination with the core genome and use small RNA genes as attachment sites for integration. We have found 13 copies of integrase genes in the Y11 genome (strain 8081 has 21 copies), but most of them seem to be truncated and no more functional. In Y11, 5 of the 13 annotated integrase genes are located next to tRNA genes. This was also the case for a tRNA-Asn that has acquired a novel genomic island of $14.9 \mathrm{kbp}$, GIYep-01. Three different tRNA-Asn loci are found in Yersinia. One of these tRNA-Asn copies has acquired the HPI in O:8/1B [20], while the GIYep-01 island (Y11_15011-Y11_15121) occupies one of the tRNA-Asn copies in O:3/4. GIYep-01 has a GC content similar to the core genome sequence. In contrast, HPI has an elevated GC content and an inactivated integrase [42]. Translated CDSs of GIYep-01 show homologies to a metallo-beta-lactamase domain containing protein, $\mathrm{SbcC}$, a protease like protein, an antirestriction protein and transition helper proteins, with the latter ones as typical members of mobile elements. To prove the mobility of GIYep-01 and functionality of its integrase, we have performed a nested PCR with JB470 and JB472, JB471 and JB473 primers to follow the restoration of the attP recombination site of the circular excised island. Results of the PCR and sequencing demonstrated the precise excision of the GIYep-1 island. Moreover, when the integrase and its attachment sites were analysed in two other serobiotype O:3/4 strains, Y8265 and Y5307, both harboured a full-length integrase and intact attachment sites.

\section{Prophages as main Y. enterocolitica ssp. palearctica acquisitions}

We found a filamentous prophage (Y11_09601-09661) in the Y11 genome that was highly homologous to CUS-1 of E. coli [43] and the Ypf prophage of Y. pestis [44]. In $Y$. pestis, the Ypf genome contains all functional modules needed for the assembly and production of viable phages and is suspected to play a role in $Y$. pestis virulence [44].

The PhiYep-1 prophage is absent from strain 8081, $\mathrm{O}: 8 / 1 \mathrm{~B}$; nevertheless, the infection with this prophage has been demonstrated for all three pathogenic Yersinia species [45]. However, the comparison of PhiYep-1 
prophages in two other serobiotype O:3/4 strains, Y8265 and Y5307, showed that the prophage sequence has already suffered successive deletions in both strains (Figure 6).

We found that the PhiYep-1 prophage constitutes a part of the $28 \mathrm{kbp}$ tandem repeat amplified in $Y$. enterocolitica after elevation of ampicillin levels [46]. This repeat harbours the blaA gene and at least part of the PhiYep-1 prophage, indicating a possible link between PhiYep-1 tandem multiplication and elevated ampicillin resistance. By PCR we proved the multiplication of the $28 \mathrm{kbp}$ fragment in Y11, but also in the absence of ampicillin. Moreover, when we raised the ampicillin concentration from $100 \mu \mathrm{g} / \mathrm{ml}$ to $1,000 \mu \mathrm{g} / \mathrm{ml}$, both for Y11 and Y5307 that lacks most of the PhiYep-1 sequence, the strains were able to grow in $1,000 \mu \mathrm{g} / \mathrm{ml}$ ampicillin containing LB media with similar overnight densities. Thus, the tandem multiplication of the PhiYep-1 prophage together with the nearby blaA region, seems not to be the only mechanism of the rapid ampicillin resistance acquisition in $\mathrm{O}: 3 / 4$.

At least two highly homologous P2-like prophages, PhiYep-2 (Y11_25141-25551) and PhiYep-3 (Y11_13081Y11_13511) are integrated in different tRNA genes in the Y11 genome; PhiYep-2 in tRNA-Met and PhiYep-3 in tRNA-Leu. These phages are highly homologous to the P2-like prophage in Y. pseudotuberculosis IP32953 (additional file 4). PhiYep-3 contains a full-length integrase and was proven for its ability to leave its attachment site. The JB606 and JB608 primers designed for restoration of the $123 \mathrm{bp}$ attP attachment site

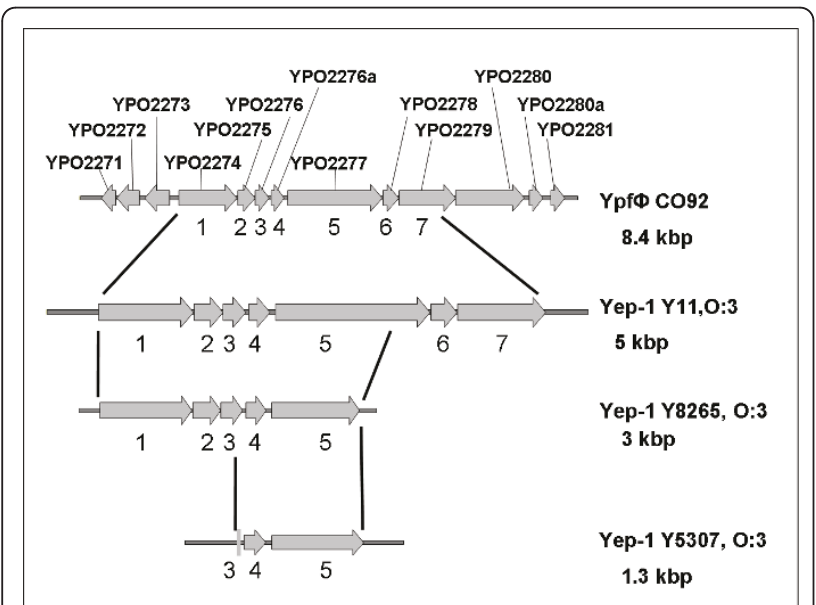

Figure 6 Comparison of the $Y$. pestis Ypf prophage and the PhiYep-1 prophage in $Y$. enterocolitica 0:3/4. The Ypf prophage is representing a typical structure of a filamentous phage, with genes for morphogenesis, secretion and replication. The cluster in $0: 3 / 4$ is notably reduced, and in strain Y5307 the Yep prophage is reduced to only three truncated genes left, as proven by sequencing demonstrated a high frequency precise excision of PhiYep-3. Nevertheless, this prophage was absent in two other serobiotype O:3/4 strains, Y8265 and Y5307. To address dissemination of PhiYep-3 in serobiotype O:3/4 strains, we performed PCR for its presence in tRNA-Leu target site. In six out of fifteen O:3/4 strains tested, the tRNA-Leu gene was occupied by the PhiYep-3 prophage (data not shown). Thus, presence of the highly active PhiYep-3 prophage may serve as an additional epidemiological marker of recent and perhaps repeated prophage acquisitions.

\section{IS-elements}

IS element copy numbers in the Yersinia genus vary between 12 and 1,147 [47]. The number of IS elements identified in Y11 genome using the ISfinder [48] was slightly higher than that in 8081 (about 64 full length IS elements). Interestingly, the variety of IS element families was higher in $\mathrm{O}: 8 / 1 \mathrm{~B}$ and includes IS 4 and IS200 family members that are absent from $0: 3 / 4$. ISYen1 is the most frequent IS element in Y11 (more than 50 copies, see Table 2).

One of the ISYen1 elements located in the promoter region of inv effects its regulation (Frank Uliczka et al. 2011, manuscript accepted by PLoS Pathogens). Furthermore, many other genes found in $\mathrm{O}: 3 / 4$ in the vicinity of ISYen1 or other IS elements can influence their activity. Examples are $c b p A$, yidE, a formate efflux transporter and dehydrogenase, $\operatorname{argO}, y c a D$, and putative virulence factors (e.g. a toxin subunit S1 precursor).

Beside the large group of ISYen1 elements, we found seven copies of the $Y$. enterocolitica ssp. palearctica specific IS-element ISYen2 [49]. This IS element is related to those of the IS21 family and its two isoforms (ISYen2A/B) are present in seven genomic copies in $\mathrm{O}: 3 / 4$. A further ISYen2B copy is located in the pYVO3 virulence plasmid [49]. We have detected at least one ISYen2A/B copy also in the O:5,27/3 genome.

\section{Discussion}

Two parallel processes, gene loss and acquisition, shape the $Y$. enterocolitica ssp. palearctica serobiotype O:3/4 genome. As expected, $Y$. enterocolitica ssp. palearctica strains do not carry the already defined high-

Table 2 Insertion sequence elements found in $Y$. enterocolitica ssp. palearctica Y11, 0:3/4.

\begin{tabular}{ll}
\hline $\begin{array}{l}\text { IS } \\
\text { element }\end{array}$ & $\begin{array}{l}\text { Number of insertion elements found in the Y11 } \\
\text { chromosome }\end{array}$ \\
\hline ISYen2A/B & 7 \\
\hline IS1400 & 1 \\
\hline ISYen4 & 1 \\
\hline ISYal1 & 2 \\
\hline ISYen1 & 53 \\
\hline
\end{tabular}


pathogenicity associated features of $8081 \mathrm{O}: 8 / 1 \mathrm{~B}$ ([50]). This decreased pathogenicity may supply the $0: 3 / 4$ group with a better chance to balance its interactions with the host and support further dissemination. Instead, $Y$. enterocolitica ssp. palearctica demonstrates an alternative pattern of putative virulence associated determinants including an RtxA-like toxin, a dual functional insecticidal toxin, beta-fimbriae and a novel $y s p$ type 3 secretion system. Since serobiotype 0:3/4 has adapted to a very narrow and specific niche, the pig tonsils, the bacteriocin cluster found in $\mathrm{O}: 3 / 4$ would also provide a serious advantage in colonisation. Likewise, the ability to utilise GalNAc of the gut mucin may represent both a virulence and fitness factor of particular importance for $\mathrm{O}: 3 / 4$ and reflects its adaptation/ association with its host.

The $y s p$ T3SS of O:3/4 that substitutes the $y s a$ T3SS of $\mathrm{O}: 8 / 1 \mathrm{~B}$ is analogous to T3SS systems of high-pathogenic $Y$. pestis and $Y$. pseudotuberculosis, indicating a potential role in pathogenicity. The effectors of this T3SS have to be identified. The $y s p$ and $y s a$ secretion systems are known to be involved both in "cross-talk" with other bacteria and with the host and they are able to support transport of "heterologous" effector molecules $[51,52]$. Thus, the ysp system might supply $Y$. enterocolitica ssp. palearctica with an additional advantage to subvert foreign imported effectors for its benefit even in the absence of the native ones.

Mobile genetic elements encoding multiple physiological traits play a significant role in bacterial evolution. A novel GIYep-01 genomic island that encodes a putative metallo-beta-lactamase and a protease in O:3/4 might be involved both in fitness and pathogenicity of yersiniae. For its integration, GIYep-01 utilizes a P4-like integrase like the HPI in $\mathrm{O}: 8 / 1 \mathrm{~B}$. However, in contrast to the HPI that is frozen to a single tRNA-Asn site in O:8/ $1 \mathrm{~B}$, the GIYep-01 can leave its initial tRNA-Asn locus due to the activity of the functional integrase. Whether the integrase of one mobility element can affect the recombination of another one and the putative role of the GIYep-01 in $Y$. enterocolitica has still to be clarified.

The filamentous PhiYep-1 prophage of O:3/4 demonstrates a high similarity to $Y$. pestis $\mathrm{Ypf}$ and $E$. coli CUS1 prophages. Both Ypf and CUS-1 are suspected to play a role in pathogenicity [43]. However, due to severe sequential deletions in the sequenced $O: 3 / 4$ strains, a possible impact of this prophage on $0: 3 / 4$ pathogenicity and elevated ampicillin resistance remains questionable.

Two copies of the highly similar P2-like prophages are present in the Y11 genome. PhiYep-2, the one with a truncated integrase, is frozen in tRNA-Met while PhiYep-3, harbouring an active integrase, is integrated into tRNA-Leu. Anyhow, the genetically active PhiYep-3 is present only in about $40 \%$ of the O:3/4 strains tested.
Thus, the PhiYep-3 prophage seems to represent a more recent Y11 acquisition and might serve as an additional epidemiological marker for $Y$. enterocolitica ssp. palearctica. The coexistence and immunity to superinfection of these two closely related P2-like prophages poses an additional question to be answered.

The presence of multiple IS elements tells a story of $Y$. enterocolitica interactions with its biotic neighbours. Indeed the spectrum of IS elements differs in $Y$. enterocolitica ssp. enterocolitica and $Y$. enterocolitica ssp. palearctica, with ISYen2A/B being the low -pathogenicity specific insertion sequence whilst a wide variety of IS families IS3, IS4 and IS200 dominates in the highpathogenicity group. These differences can be applied both to subspecies identification and also for tracing history of interbacterial interactions.

It is remarkable that the gene clusters with potentially closely related functions tend to occupy exactly the same positions ("hot spots") in the $Y$. enterocolitica backbone genomes (like the T3SS, the O-antigen, an AidA adhesin and haemolysin, the OspG protein kinase gene cluster, etc.). On the other hand, divergence in these clusters might result from both vertical and horizontal evolution events.

$Y$. enterocolitica ssp. palearctica O:3/4 has an open genome with traces of frequent gene import and wastes. These different vectors have formed the present $0: 3 / 4$ genome that combines standard yersinial determinants with putative alternative virulence and fitness associated factors. Such a patchwork seems to be a prerequisite for $\mathrm{O}: 3 / 4$ successful worldwide dissemination.

\section{Conclusions}

$Y$. enterocolitica ssp. palearctica $\mathrm{O}: 3 / 4$ becomes nowadays the dominating Yersinia worldwide. Multiple gene losses and acquisitions experienced by its genome shift this pathogen to explore an alternative patchwork of virulence and fitness determinants for the efficient proliferation. Future analysis of these combinations of different virulence traits may shed a new light on the processes and structures behind successful dissemination of bacterial pathogens in the modern world in general and of the successful emerging zoonotic pathogen $Y$. enterocolitica ssp. palearctica $\mathrm{O}: 3 / 4$ in particular.

\section{Methods}

\section{Strains and DNA preparation}

The $Y$. enterocolitica ssp. palearctica Y11 (DSMZ type strain no. 13030) serobiotype O:3/4 strain isolated from human stool was selected for complete genome sequencing. Strain Y8265 (derived from a human isolate, France; [53]) and Y5307 (derived from a reactive arthritis patient, institute's strain collection) of the same serobiotype $\mathrm{O}: 3 / 4$ and strain Y5,27P of serobiotype O:5,27/3 
(institute's strain collection) were used for high coverage draft genome sequencing (see below). DNA was prepared using NucleoBond ${ }^{\circledR}$ AXG of Macherey-Nagel (Düren, Germany) following the manufacturers instructions.

For $\mathrm{N}$-acetyl-galactosamine uptake experiments, the human isolate strain WA-314 O:8/1B was used [53].

\section{Genome sequencing and bioinformatics}

The genome sequence of $Y$. enterocolitica ssp. palearctica O:3/4 strain Y11 was determined by combination of high-throughput whole genome shotgun sequencing using MegaBACE (at Integrated Genomics, Jena, Germany), 454 Genome Sequencer (GS) 20 (at 454, Branford CT, USA) and an additional 454 GS FLX Titanium run (at Seq-IT, Kaiserslautern, Germany). Gaps were closed manually in cooperation with LGC Genomics (Berlin, Germany) by PCR followed by Sanger sequencing of the respective amplification products. The last gap that constituted a second copy of a highly homologous P2-like prophage was closed by primer walking on a single phage-spanning fosmid clone. Finally, the raw data were assembled using the Newbler software (454 Life Sciences Corporation, Version Software Release 2.3) into a complete genome sequence of 4,553,420 bp for the genome and an additional 72,463 bp contig representing the pYVO3 plasmid. The complete genome sequence of this strain has been published recently [17]. The draft high coverage genome sequences of three other $Y$. enterocolitica ssp. palearctica strains of human origin, Y8265 and Y5307 of serobiotype O:3/4 and Y5,27P of serobiotype O:5,27/3 were obtained in cooperation with BGI-Hongkong Co., Hong Kong. We used high-throughput Illumina sequencing technology to conduct paired-end sequencing for DNA samples, and constructed a $500 \mathrm{bp}$ library with extended data of $500 \mathrm{Mb}$, and a $6 \mathrm{kbp}$ library with expected data of $250 \mathrm{Mb}$. Genome assembly results in 14 large scaffolds and 215 contigs for Y8265, 18 scaffolds and 256 contigs for Y5307, and 20 scaffolds and 408 contigs for the Y5,27P strain. Genome coverage based on k-mer exceeds 105\%, and genome coverage based on reads mapping was $97 \%$ for the three genomes. The genome sequences were annotated using the RAST server [54]. Genome comparisons have been done using SEED [55], the Artemis comparison tool [56], Mauve [57] and other standard comparison tools. SEED was also used to determine orthologous proteins, using the standard parameters.

Accession numbers of strains used in this study are listed in Table 3.

\section{Excision of genetic mobile elements}

The excision of GIYep-01 island was verified by nested PCR amplifying the attP attachment sites of the circular
Table 3 Accession numbers of strains involved in this study

\begin{tabular}{ll}
\hline $\begin{array}{l}\text { Strain name and } \\
\text { bioserotype }\end{array}$ & $\begin{array}{l}\text { Accession numbers in EMBL } \\
\text { database }\end{array}$ \\
\hline Y11, O:3/4 & FR729477 and FR745874 (plasmid) \\
\hline 8081, O:8/1B & NC_008800 and NC_008791 (plasmid) \\
\hline 105.5R(r), O:9/3 & CP002246 and CP002247 (plasmid) \\
\hline W22703, O:9/2 & AF102990 (plasmid) \\
\hline Y8265, O:3/4 & CACU01000001-CACU01000014 \\
\hline Y5307, O:3/4 & CACV01000001-CACV01000018 \\
\hline Y5,27P, O:5,27/3 & CACW01000001-CACW01000020 \\
\hline
\end{tabular}

excised element (the second PCR covering about $420 \mathrm{bp}$ ) followed by subsequent sequencing. The following oligonucleotides have been used (bold letters, reverse orientation oligonucleotides): JB470, agaatcggaaactttgaatggttt, JB472, CACATCAGGCACTTCTCCAGG, and JB 471, ttgagccgttaagagacatttgg, and JB473, TTAACAGAAATAG CGCCCAT.

In the case of the PhiYep-3 excision, a single PCR amplification step was sufficient for amplification of the attP attachment site of the circular excised prophage (about $500 \mathrm{bp}$, proven by subsequent sequencing of the PCR product). For PhiYep-3, the following oligonucleotides have been used: JB606, GGCGTGTTGTGGATGTAAT and JB608, atgtcagtatatttggcgat. For PhiYep-3 dissemination analysis, 15 strains of serobiotype $\mathrm{O}: 3 / 4$ have been tested.

\section{$\mathrm{N}$-acetyl-galactosamine experiments}

The 8.4 kbp aga-operon (Y11_11961-Y11_12031) has been subcloned into pGEM-T Easy (Promega) using the following oligonucleotides: JB506 cagcgtcgtacttgatgatttgc and JB507 ATCATCTGTTGGGCGACACG. The aga-supplemented serobiotype O:8/1B strain was grown in the presence of carbenicillin $(300 \mu \mathrm{g} / \mathrm{ml})$ to maintain the plasmid, serobiotype $\mathrm{O}: 3 / 4$ and $\mathrm{O}: 8 / 1 \mathrm{~B}$ wild type strains were cultivated without antibiotics. M9 minimal medium supplemented with $1 \mathrm{mM}$ $\mathrm{MgSO}_{4}$ and $0.1 \mathrm{mM} \mathrm{CaCl}_{2}$ and the appropriate amino sugars $(0.2 \%$ of $\mathrm{N}$-acetyl-galactosamine, $\mathrm{N}$-acetyl-glucosamine, glucose and galactose) was used in all experiments. Tryptophan (Trp) was added $(200 \mu \mathrm{g} / \mathrm{ml})$ to support sufficient Yersinia enterocolitica growth. The bacteria were grown in $0.01 \mathrm{M}$ glucose supplemented M9 overnight (16-20 hours) as a preculture, pelleted and washed with M9 without sugar additives. The optical density was measured and all samples were inoculated to the same $\mathrm{OD}_{600}$ 0.2-0.3. Experiments were carried out in 10-20 ml M9 in $50 \mathrm{ml}$ Falcon tubes. The optical density of the cultures was measured after inoculation and 48 hours. 


\section{Further particulars}

The authors declare that they have no competing interests.

\section{Additional material}

\section{Additional file 1: Additional table with general CDS features of serobiotypes $0: 3 / 4$ and $0: 8 / 1 \mathrm{~B}$ \\ Additional file 2: Additional Excel table with all protein comparisons (including locus tags) of the annotated genome of strain Y11 with related genomes (serobiotype 0:5,27/3; two additional serobiotype 0:3/4 strains; serobiotype O:8/1B strain 8081).}

Additional file 3: Additional figure with a comparison of the pYV plasmid of serobiotypes 0:3/4, 0:9/2 (pYVe227) and 0:8/1B using the Artemis Comparison Tool (ACT).

Additional file 4: Additional figure with a comparison of the genome sequence of the two P2-like prophages PhiYep-2 and PhiYep-3 with the homologous P2-like prophage in $Y$. pseudotuberculosis IP 32953

\section{Acknowledgements}

This work was supported by the German Bundesministerium für Bildung und Forschung (BMBF) Network Grant FBI-Zoo [01KI07122] in both experimental work and manuscript preparation. The German National Network of FoodBorne Zoonotic Infections of Humans, FBI-Zoo, focuses on characterization, (sub-) typing, and epidemiological analysis of zoonotic food borne pathogens, including $Y$. enterocolitica. Also the mechanisms for successful exploration of the established and new ecological niches are addressed and analysed. Our present work influenced by this epidemiological view aims to shed a new light onto the differences between the closely related $Y$. enterocolitica subspecies on the genomic level.

We are thankful to C. Batzilla and O. Podladchikova for helpful discussions and reviewing the manuscript. Further we appreciate the work of our former colleague A. Golubov for this genome project as well as the fruitful discussions with $\mathrm{H}$. Neubauer.

\section{Author details}

${ }^{1}$ Max von Pettenkofer-Institute, LMU, Munich, Germany. ${ }^{2}$ Institute of Diagnostic Virology, Friedrich-Loeffler-Institute, Greifswald-Insel Riems, Germany.

\section{Authors' contributions}

JB has carried out the main genome comparisons, designed and executed all experiments, participated in the gap closure of the genome sequence and revised the sequence. JB also wrote major parts of the manuscript and designed figures and tables. UA has done further gene comparisons, gave setup advice for the sugar uptake experiments and participated in the composition of the manuscript. DH participated in the genome sequence alignment, conducted the quality control of the assembled raw data and added helpful ideas to the manuscript. JH and AR have planned and supervised the experiments and genome comparisons, and AR has written major parts of the introduction and discussion. All authors read and approved the final manuscript.

Received: 10 January 2011 Accepted: 6 July 2011 Published: 6 July 2011

\section{References}

1. Wren BW: The yersiniae-a model genus to study the rapid evolution of bacterial pathogens. Nat Rev Microbiol 2003, 1:55-64.

2. Achtman M, Zurth K, Morelli G, Torrea G, Guiyoule A, Carniel E: Yersinia pestis, the cause of plague, is a recently emerged clone of Yersinia pseudotuberculosis. Proc Natl Acad Sci USA 1999, 96:14043-14048.

3. Wauters G: [Pathogenic and epidemiologic aspects of Yersinia enterocolitica infections]. Bull Mem Acad R Med Belg 1981, 136:510-520.
4. Wauters G, Kandolo K, Janssens M: Revised biogrouping scheme of Yersinia enterocolitica. Contrib Microbiol Immunol 1987, 9:14-21.

5. Rosner BM, Stark K, Werber D: Epidemiology of reported Yersinia enterocolitica infections in Germany, 2001-2008. BMC Public Health 2010, 10:337.

6. Bonardi S, Brindani F, Pizzin G, Lucidi L, D'Incau M, Liebana E, Morabito S: Detection of Salmonella spp., Yersinia enterocolitica and verocytotoxinproducing Escherichia coli $\mathrm{O} 157$ in pigs at slaughter in Italy. Int J Food Microbiol 2003, 85:101-110.

7. Martinez PO, Fredriksson-Ahomaa M, Pallotti A, Rosmini R, Houf K, Korkeala $\mathrm{H}$ : Variation in the Prevalence of Enteropathogenic Yersinia in Slaughter Pigs from Belgium, Italy, and Spain. Foodborne Pathog Dis 2010.

8. Bottone EJ: Yersinia enterocolitica: the charisma continues. Clin Microbiol Rev 1997, 10:257-276.

9. Fredriksson-Ahomaa $\mathrm{M}$, Stolle $\mathrm{A}$, Korkeala $\mathrm{H}$ : Molecular epidemiology of Yersinia enterocolitica infections. FEMS Immunol Med Microbiol 2006, 47:315-329.

10. Fredriksson-Ahomaa $M$, Stolle $A$, Siitonen A, Korkeala H: Sporadic human Yersinia enterocolitica infections caused by bioserotype 4/0:3 originate mainly from pigs. J Med Microbiol 2006, 55:747-749.

11. Fredriksson-Ahomaa M, Niskanen $T$, Bucher M, Korte $T$, Stolle A, Korkeala H: Different Yersinia enterocolitica 4:O3 genotypes found in pig tonsils in Southern Germany and Finland. Syst Appl Microbiol 2003, 26:132-137.

12. Fredriksson-Ahomaa M, Korkeala H: Molecular epidemiology of Yersinia enterocolitica 4/O:3. Adv Exp Med Biol 2003, 529:295-302.

13. Bucher M, Meyer C, Grotzbach B, Wacheck S, Stolle A, FredrikssonAhomaa M: Epidemiological data on pathogenic Yersinia enterocolitica in Southern Germany during 2000-2006. Foodborne Pathog Dis 2008, 5:273-280.

14. Laukkanen R, Martinez PO, Siekkinen KM, Ranta J, Maijala R, Korkeala H: Contamination of carcasses with human pathogenic Yersinia enterocolitica 4/0:3 originates from pigs infected on farms. Foodborne Pathog Dis 2009, 6:681-688.

15. Thomson NR, Howard S, Wren BW, Holden MT, Crossman L, Challis GL, Churcher C, Mungall K, Brooks K, Chillingworth T, et al: The complete genome sequence and comparative genome analysis of the high pathogenicity Yersinia enterocolitica strain 8081. PLoS Genet 2006, 2:e206.

16. Neubauer H, Aleksic S, Hensel A, Finke EJ, Meyer H: Yersinia enterocolitica 16S rRNA gene types belong to the same genospecies but form three homology groups. Int J Med Microbiol 2000, 290:61-64.

17. Batzilla J, Hoper D, Antonenka U, Heesemann J, Rakin A: Complete genome sequence of Yersinia enterocolitica subsp. palearctica serogroup 0:3. J Bacteriol 2011

18. Rakin A, Noelting C, Schubert S, Heesemann J: Common and specific characteristics of the high-pathogenicity island of Yersinia enterocolitica. Infect Immun 1999, 67:5265-5274.

19. Pelludat $C$, Hogardt $M$, Heesemann J: Transfer of the core region genes of the Yersinia enterocolitica WA-C serotype O:8 high-pathogenicity island to $Y$. enterocolitica MRS40, a strain with low levels of pathogenicity, confers a yersiniabactin biosynthesis phenotype and enhanced mouse virulence. Infect Immun 2002, 70:1832-1841.

20. Carniel E, Guilvout I, Prentice M: Characterization of a large chromosomal "high-pathogenicity island" in biotype 1B Yersinia enterocolitica. J Bacteriol 1996, 178:6743-6751.

21. Haller JC, Carlson S, Pederson KJ, Pierson DE: A chromosomally encoded type III secretion pathway in Yersinia enterocolitica is important in virulence. Mol Microbiol 2000, 36:1436-1446.

22. Skurnik M, Bengoechea JA: The biosynthesis and biological role of lipopolysaccharide O-antigens of pathogenic Yersiniae. Carbohydr Res 2003, 338:2521-2529.

23. Skurnik M: Molecular genetics, biochemistry and biological role of Yersinia lipopolysaccharide. Adv Exp Med Biol 2003, 529:187-197.

24. Welch RA: RTX toxin structure and function: a story of numerous anomalies and few analogies in toxin biology. Curr Top Microbiol Immunol 2001, 257:85-111.

25. Linhartova I, Bumba L, Masin J, Basler M, Osicka R, Kamanova J, Prochazkova K, Adkins I, Hejnova-Holubova J, Sadilkova L, et al: RTX proteins: a highly diverse family secreted by a common mechanism. FEMS Microbiol Rev 2010, 34:1076-1112.

26. Lally ET, Hill RB, Kieba IR, Korostoff J: The interaction between RTX toxins and target cells. Trends Microbiol 1999, 7:356-361. 
27. Kudryashov DS, Durer ZA, Ytterberg AJ, Sawaya MR, Pashkov I, Prochazkova K, Yeates TO, Loo RR, Loo JA, Satchell KJ, Reisler E: Connecting actin monomers by iso-peptide bond is a toxicity mechanism of the Vibrio cholerae MARTX toxin. Proc Natl Acad Sci USA 2008, 105:18537-18542.

28. Kudryashov DS, Cordero CL, Reisler E, Satchell KJ: Characterization of the enzymatic activity of the actin cross-linking domain from the Vibrio cholerae MARTX Vc toxin. J Biol Chem 2008, 283:445-452.

29. Felmlee T, Pellett $S$, Welch RA: Nucleotide sequence of an Escherichia coli chromosomal hemolysin. J Bacteriol 1985, 163:94-105.

30. Felmlee T, Pellett S, Lee EY, Welch RA: Escherichia coli hemolysin is released extracellularly without cleavage of a signal peptide. J Bacteriol 1985, 163:88-93.

31. Kasari V, Kurg K, Margus T, Tenson T, Kaldalu N: The Escherichia coli mqsR and ygiT genes encode a new toxin-antitoxin pair. J Bacteriol 2010, 192:2908-2919.

32. Garcia-Contreras R, Zhang XS, Kim Y, Wood TK: Protein translation and cell death: the role of rare tRNAs in biofilm formation and in activating dormant phage killer genes. PLoS One 2008, 3:e2394.

33. Bresolin G, Morgan JA, Ilgen D, Scherer S, Fuchs TM: Low temperatureinduced insecticidal activity of Yersinia enterocolitica. Mol Microbio/ 2006, 59:503-512.

34. Fuchs TM, Bresolin G, Marcinowski L, Schachtner J, Scherer S: Insecticidal genes of Yersinia spp.: taxonomical distribution, contribution to toxicity towards Manduca sexta and Galleria mellonella, and evolution. BMC Microbiol 2008, 8:214.

35. Bresolin G, Trcek J, Scherer S, Fuchs TM: Presence of a functional flagellar cluster Flag-2 and low-temperature expression of flagellar genes in Yersinia enterocolitica W22703. Microbiology 2008, 154:196-206.

36. Nuccio SP, Baumler AJ: Evolution of the chaperone/usher assembly pathway: fimbrial classification goes Greek. Microbiol Mol Biol Rev 2007, 71:551-575.

37. Waterman SR, Holden DW: Functions and effectors of the Salmonella pathogenicity island 2 type III secretion system. Cell Microbiol 2003, 5:501-511

38. Nishi J, Sheikh J, Mizuguchi K, Luisi B, Burland V, Boutin A, Rose DJ, Blattner FR, Nataro JP: The export of coat protein from enteroaggregative Escherichia coli by a specific ATP-binding cassette transporter system. J Biol Chem 2003, 278:45680-45689.

39. Mantle M, Pearson J, Allen A: Pig gastric and small-intestinal mucus glycoproteins: proposed role in polymeric structure for protein joined by disulphide bridges. Biochem Soc Trans 1980, 8:715-716

40. Forstner JF, Jabbal I, Qureshi R, Kells DI, Forstner GG: The role of disulphide bonds in human intestinal mucin. Biochem J 1979, 181:725-732.

41. Delpino MV, Marchesini MI, Estein SM, Comerci DJ, Cassataro J, Fossati CA, Baldi PC: A bile salt hydrolase of Brucella abortus contributes to the establishment of a successful infection through the oral route in mice. Infect Immun 2007, 75:299-305.

42. Bach S, Buchrieser C, Prentice M, Guiyoule A, Msadek T, Carniel E: The highpathogenicity island of Yersinia enterocolitica Ye8081 undergoes lowfrequency deletion but not precise excision, suggesting recent stabilization in the genome. Infect Immun 1999, 67:5091-5099.

43. Gonzalez MD, Lichtensteiger CA, Caughlan R, Vimr ER: Conserved filamentous prophage in Escherichia coli 018:K1:H7 and Yersinia pestis biovar orientalis. J Bacteriol 2002, 184:6050-6055.

44. Derbise A, Chenal-Francisque V, Pouillot F, Fayolle C, Prevost MC, Medigue C, Hinnebusch BJ, Carniel E: A horizontally acquired filamentous phage contributes to the pathogenicity of the plague bacillus. $\mathrm{Mol}$ Microbiol 2007, 63:1145-1157.

45. Chouikha I, Charrier L, Filali S, Derbise A, Carniel E: Insights into the infective properties of YpfPhi, the Yersinia pestis filamentous phage. Virology 2010, 407:43-52

46. Seoane A, Sanchez E, Garcia-Lobo JM: Tandem amplification of a 28kilobase region from the Yersinia enterocolitica chromosome containing the blaA gene. Antimicrob Agents Chemother 2003, 47:682-688.

47. Chen PE, Cook C, Stewart AC, Nagarajan N, Sommer DD, Pop M, Thomason B, Thomason MP, Lentz S, Nolan N, et al: Genomic characterization of the Yersinia genus. Genome Biol 2010, 11:R1.

48. Siguier P, Perochon J, Lestrade L, Mahillon J, Chandler M: ISfinder: the reference centre for bacterial insertion sequences. Nucleic Acids Res 2006, 34:D32-36.
49. Golubov A, Gierczynski R, Heesemann J, Rakin A: A novel insertion sequence element, IS Yen2, as an epidemiological marker for weakly pathogenic bioserotypes of Yersinia enterocolitica. Int J Med Microbiol 2005, 295:213-226.

50. Howard SL, Gaunt MW, Hinds J, Witney AA, Stabler R, Wren BW: Application of comparative phylogenomics to study the evolution of Yersinia enterocolitica and to identify genetic differences relating to pathogenicity. J Bacteriol 2006, 188:3645-3653.

51. Young BM, Young GM: Evidence for targeting of Yop effectors by the chromosomally encoded Ysa type III secretion system of Yersinia enterocolitica. J Bacteriol 2002, 184:5563-5571.

52. Young BM, Young GM: YplA is exported by the Ysc, Ysa, and flagellar type III secretion systems of Yersinia enterocolitica. J Bacteriol 2002, 184:1324-1334

53. Saken E, Roggenkamp A, Aleksic S, Heesemann J: Characterisation of pathogenic Yersinia enterocolitica serogroups by pulsed-field gel electrophoresis of genomic Notl restriction fragments. J Med Microbiol 1994, 41:329-338.

54. Aziz RK, Bartels D, Best AA, DeJongh M, Disz T, Edwards RA, Formsma K, Gerdes S, Glass EM, Kubal M, et al: The RAST Server: rapid annotations using subsystems technology. BMC Genomics 2008, 9:75

55. Overbeek R, Begley T, Butler RM, Choudhuri JV, Chuang HY, Cohoon M, de Crecy-Lagard V, Diaz N, Disz T, Edwards R, et al: The subsystems approach to genome annotation and its use in the project to annotate 1000 genomes. Nucleic Acids Res 2005, 33:5691-5702.

56. Carver TJ, Rutherford KM, Berriman M, Rajandream MA, Barrell BG, Parkhill J: ACT: the Artemis Comparison Tool. Bioinformatics 2005, 21:3422-3423.

57. Darling AE, Mau B, Perna NT: progressiveMauve: multiple genome alignment with gene gain, loss and rearrangement. PLoS One 2010, 5 e11147.

doi:10.1186/1471-2164-12-348

Cite this article as: Batzilla et al:: Yersinia enterocolitica palearctica serobiotype 0:3/4 - a successful group of emerging zoonotic pathogens. BMC Genomics 2011 12:348.

\section{Submit your next manuscript to BioMed Central and take full advantage of:}

- Convenient online submission

- Thorough peer review

- No space constraints or color figure charges

- Immediate publication on acceptance

- Inclusion in PubMed, CAS, Scopus and Google Scholar

- Research which is freely available for redistribution

Submit your manuscript at www.biomedcentral.com/submit
C) Biomed Central 